\title{
Presencia de Cotylophoron sp en Bovinos de la Provincia de Moyobamba, Perú
}

\author{
Presence of Cotylophoron sp in Bovine of the Province of Moyobamba, Peru \\ Kelwin Rojas H. ${ }^{1}$, Enrique Serrano-Martínez ${ }^{1,2}$, Manuel Tantaleán V. ${ }^{1}$, \\ Gina C. Casas V. ${ }^{1}$, Marco Quispe H. ${ }^{1}$
}

\section{Resumen}

El objetivo del presente estudio fue determinar la presencia de Cotylophorum sp en ganado bovino lechero en seis distritos de la provincia de Moyobamba, Perú. Se recolectaron 411 muestras de heces de bovinos con edades de 1.5 a 8 años, entre octubre y noviembre de 2011, y se procesaron mediante el método de Sedimentación Rápida. Los resultados determinaron la presencia de huevos de Cotylophoron sp con una prevalencia de 55\%. No se evidenció diferencia estadística significativa entre las variables edad, sexo y lugar de procedencia y la presencia de Cotylophoron sp.

Palabras clave: trematodos, bovino, Cotylophoron, sedimentación, Moyobamba

\section{Abstract}

The aim of this study was to determine the presence of Cotylophorum sp in cattle in six districts of the province of Moyobamba, Peru. A total of 411 faecal samples of cattle aged 1.5 to 8 years between October and November 2011 were collected. The samples were processed by the method of feacal sedimentation. The results showed the presence of eggs of Cotylophoron sp, with a prevalence of 55\%. No statistically difference was found between the variables age, sex and place of origin and the presence of Cotylophoron sp.

Key words: flukes, bovine, Cotylophoron, sedimentation, Moyobamba

\footnotetext{
${ }^{1}$ Grupo SALUVET-UPCH, Facultad de Medicina Veterinaria y Zootecnia, Universidad Peruana Cayetano Heredia, Lima, Perú

${ }^{2}$ E-mail: enrique.serrano@upch.pe

Recibido: 5 de marzo de 2014

Aceptado para publicación: 28 de abril de 2015
} 


\section{INTRODUCCIÓN}

La paramfistomosis es una enfermedad parasitaria ocasionada por trematodos que afectan a rumiantes (bovinos, ovinos), porcinos y diversas especies silvestres, especialmente en regiones tropicales (Sánchez et al., 2009). Esta parasitosis involucra géneros como Paramphistomum, Cotylophoron, Calicophoron, Ugandocotyle, Orthocoelium y Balanorchis (Eduardo, 1982). En el Perú se consideró por varios años a Paramphistomum cervi (Tantaleán et al., 1992); sin embargo, estudios recientes refieren que el principal paramfistómido presente es Cotylophorum cotylophorum, especie ampliamente distribuida en el continente americano (Sánchez et al., 2009).

Los paramfistómidos se caracterizan por tener el cuerpo convexo dorsalmente, con un espesor de 2 a $5 \mathrm{~mm}$ y cerca de $1 \mathrm{~cm}$ de largo. Presentan una ventosa ventral denominada acetábulo en posición terminal y una ventosa oral que se encuentra en el polo anterior que es más pequeña; asimismo, carecen de espinas en el cuerpo, las cuales son reemplazadas frecuentemente por papilas tegumentarias (Barriga, 2002).

Los estadios adultos están presentes generalmente en el rumen y raramente en omaso y abomaso del hospedero definitivo, el rumiante (Dirksen et al., 2005). Los huevos son eliminados con las heces, donde se desarrolla el miracidio, el cual penetra en un caracol acuático pulmonado, mayormente de los géneros Bulinus, Planorbis y de la familia Lymnaeidae (Soulsby, 1987). En el hospedero intermediario se desarrollan los estadios de miracidio, esporocisto, redias y cercarias. En el medio ambiente se enquistan formando la metacercaria, estadio infectivo del parásito. El desarrollo de las metacercarias en el hospedero definitivo ocurre en el tracto digestivo (Percedo y Larrmendy, 1989; Abrous et al., 1999).
Los trastornos clínicos producidos por los vermes adultos fijados a la mucosa del rumen son menores que los originados por las fases juveniles migrantes, que ocasionan diarrea fétida y profusa, anorexia, pérdida de peso e incluso la muerte (Soulsby, 1987). El diagnóstico se basa en los signos clínicos, la historia de exposición a pastos con posible contaminación parasitaria y la presencia de huevo de trematodos en las heces diarreicas (Urquhart et al., 2001; Barriga, 2002). Es importante establecer el diagnóstico diferencial frente a otros trematodos, especialmente con Fasciola hepatica, dada la similitud de los huevos.

En Perú se ha reportado Paramphistomidae sp desde 1975 (Tantaleán et al., 1975) en un bovino de la región de Loreto. Trigueros (2003) notifica la presencia de paramfistómidos, sin señalar género ni especie, en un grupo de ovinos de la zona de Pucallpa con cuadros diarreicos y con ocurrencia de muertes. Posteriormente, Sánchez et al. (2009) realizaron estudios morfológicos de trematodos adultos colectados en el camal de Maynas (Loreto), señalando a $C$. cotylophorum como la principal especie de paramfistómido presente. Esta especie también ha sido notificada en Colombia, Venezuela, Brasil y Argentina (Pino y Morales, 1982; Racioppi et al., 1994; Alarcón y Velásquez, 2009).

Es así, que las prevalencias de trematodos en bovinos de diversas zonas del Perú varían desde moderadas a altas, observándose infecciones mixtas en algunos casos (Paucar et al., 2010; Torrel, 2008; Pinedo et al., 2010). Debido a esto, el objetivo del presente estudio fue determinar la posible presencia de Cotylophorum sp en ganado bovino de la provincia de Moyobamba, San Martín. 


\section{Materiales y Métodos}

\section{Lugar de Estudio y Animales}

El estudio fue realizado en predios de ganado bovino lechero ubicados en los distritos de Calzada, Habana, Jepelacio, Moyobamba, Soritor y Yantalo, provincia de Moyobamba, departamento de San Martín, Perú. La zona se encuentra entre 635 y 1113 msnm, presenta un clima húmedo y cálido, temperatura máxima promedio anual de 27 a $35^{\circ} \mathrm{C}$ y precipitación pluvial anual de 1512 mm (Municipalidad de Moyobamba, 2006).

Los animales eran del tipo criollo, entre 1.5 a 8 años, y criados en sistemas semiintensivos con fines de producción de leche. El manejo de los animales incluía un programa de desparasitación a base de albendazol.

\section{Colección y Procesamiento de Muestras}

El tamaño muestral fue calculado mediante muestreo aleatorio simple a partir de la distribución poblacional de ganado bovino de la región San Martín. Para el cálculo se aplicó la fórmula para poblaciones finitas, considerando una población de 18835 animales, según el Ministerio de Agricultura (1984). El tamaño muestral mínimo resultante fue de 411 animales.

La recolección de muestras de heces ( $n=411$ ) fue estratificada según el lugar de procedencia, y considerando el tamaño poblacional de cada distrito (INEI, 1994). Fueron muestreados 29 fundos, fluctuando entre 2 a 30 animales por fundo. El número resultante de animales muestreados según sexo fue de 55 machos y 356 hembras.

Las muestras (40-50 g) se obtuvieron directamente del recto de los animales entre octubre y noviembre de 2011 (época de lluvia) y se colocaron en frascos herméticos en cajas isotérmicas a $4{ }^{\circ} \mathrm{C}$, hasta su traslado vía aérea al Laboratorio de Parasitología de la Facultad de Veterinaria y Zootecnia de la
Universidad Peruana Cayetano Heredia, en Lima. El tiempo transcurrido entre la toma de muestra y su procesamiento fue de un máximo de 48 horas.

Se registró la fecha de muestreo, edad del animal, sexo y lugar de procedencia. El análisis de las muestras se hizo mediante el método de sedimentación rápida. Los huevos de Cotylophoron spp se identificaron por su forma ovoide, presencia de opérculo, cáscara estriada, color amarillo brillante, y medidas cercanas a $120 \times 70 \mu \mathrm{m}$, teniendo como referencia el reporte de Alarcón y Velásquez (2009).

\section{Análisis de Datos}

Los datos obtenidos fueron expresados en forma porcentual (Thrusfield, 1990). La posible asociación entre variables (edad [cuatro grupos etarios], sexo [macho, hembra], procedencia [seis distritos]) fue analizada mediante la prueba de regresión logística.

\section{Resultados y Discusión}

El presente estudio confirmó la presencia de Cotylophoron sp en el $55.0 \%$ de los animales (Fig. 1, Cuadro 1).

Las especies del género Cotylophoron afectan a rumiantes domésticos y silvestres, principalmente, en las regiones tropicales y subtropicales. Sánchez et al. (2009) demostraron en muestras obtenidas del camal de Iquitos, Perú, que la especie presente en los bovinos era $C$. cotylophorum, especie también presente en otros países sudamericanos (Pino y Morales, 1982; Raccioppi et al., 1994).

El grupo etario con mayor prevalencia fue el de 5 a 7 años (61.9\%), mientras que la variable sexo presentó mayor prevalencia en machos (63.6\%); sin embargo, estas diferencias no fueron estadísticamente significativas (Cuadro 1). 
Cuadro 1. Prevalencia de Cotylophoron sp en bovinos criollos de Moyobamba, Perú (2011)

\begin{tabular}{|c|c|c|c|c|}
\hline \multirow{2}{*}{ Variable } & & \multirow{2}{*}{ N. ${ }^{\circ}$} & \multicolumn{2}{|c|}{ Cotylophoron sp. } \\
\hline & & & $\mathrm{n}$ & $\%$ \\
\hline \multirow{6}{*}{$\begin{array}{l}\text { Lugar de } \\
\text { procedencia }\end{array}$} & Calzada & 40 & 26 & $65.0 \pm 14.8$ \\
\hline & Habana & 22 & 15 & $68.2 \pm 19.5$ \\
\hline & Jepelacio & 130 & 75 & $57.7 \pm 8.5$ \\
\hline & Moyobamba & 123 & 71 & $57.7 \pm 8.7$ \\
\hline & Soritor & 85 & 35 & $41.2 \pm 10.5$ \\
\hline & Yantalo & 11 & 4 & $36.4 \pm 28.4$ \\
\hline \multirow{4}{*}{$\begin{array}{l}\text { Grupo etario } \\
\text { (años) }\end{array}$} & 1.5 a 2 & 69 & 37 & $53.6 \pm 11.8$ \\
\hline & $>2$ a 5 & 194 & 103 & $53.0 \pm 7.0$ \\
\hline & $>5$ a 7 & 84 & 52 & $61.9 \pm 10.4$ \\
\hline & $>7$ a 8 & 64 & 34 & $53.1 \pm 12.2$ \\
\hline \multirow[t]{2}{*}{ Sexo } & Macho & 55 & 35 & $63.6 \pm 12.7$ \\
\hline & Hembra & 356 & 191 & $53.6 \pm 5.2$ \\
\hline Total & & 411 & 226 & $55.0 \pm 4.8$ \\
\hline
\end{tabular}

En relación al lugar de procedencia, se observó mayor prevalencia en los distritos de Habana y Calzada (68.2 y $65.0 \%$, respectivamente) en comparación con los demás distritos que presentaron prevalencia de 36.4 a $57.7 \%$ (Cuadro 1), pero estas diferencias no fueron estadísticamente importantes. Las diferencias observadas podrían atribuirse a la que algunas zonas tenían mayores accesos a charcos, riachuelos o zonas con mayor cantidad de hospederos intermediarios; además de un clima más favorable para su desarrollo.

Con base a los resultados hallados en el presente estudio, se presume la posibilidad de que en regiones con geografía similar al presente estudio y donde exista la convivencia simultánea de Cotylophoron con otro trematodo como Fasciola hepatica, la prevalencia de Cotylophoron se encuentre subestimada debido a que el diagnóstico de ambos parásitos en la etapa crónica de la enfermedad se realice por métodos coproparasi-

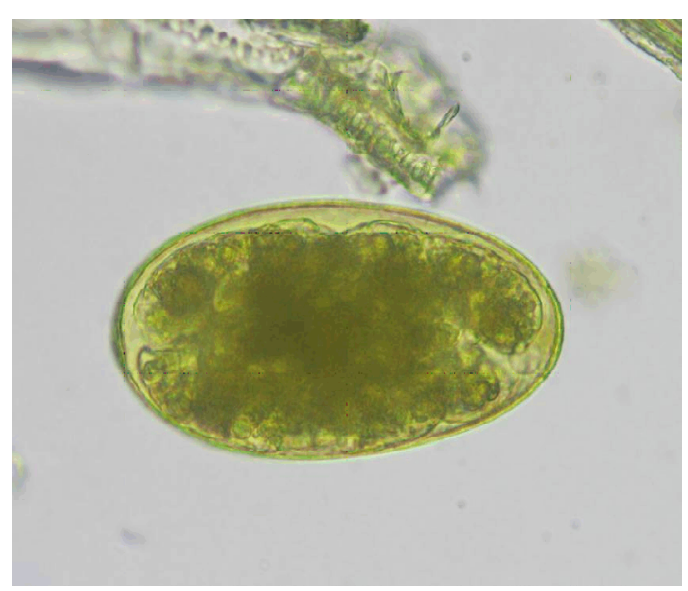

Figura 1. Huevo de Cotylophoron sp (400x) encontrado en heces de bovinos de Moyobamba, Perú

tológicos, dificultando la diferenciación de ambas especies por la similitud morfológica de los huevos, pudiendo presentarse errores en el diagnóstico (Barriga, 2002). 
La elevada prevalencia de Cotylophoron sp puede verse favorecida por desparasitaciones inadecuadas, utilizando antiparasitarios de amplio espectro como el albendazol, el cual es efectivo para el control de nematodos, pero no para el control de formas inmaduras de ciertos trematodes; especialmente para aquellos de la familia Paramphistomidae. Reportes actuales mencionan a la oxiclozanida como fármaco adecuado frente al control de los paramfistómidos (Paraud et al., 2009). Asimismo, Rojas y Torrel (2015) mencionan como tratamiento para formas inmaduras al hexaclorofeno, niclofolán, oxaclozanida, rafoxanide y febantel, y para las formas maduras al biotionol, niclosamida y resorantel.

Los resultados hallados justifican la realización de estudios epidemiológicos que permitan evaluar la presencia de las especies de paramfistómidos en el país, más aun teniendo en cuenta la similitud de las formas parasitarias que dificultan establecer un adecuado tratamiento antiparasitario en el ganado.

\section{Conclusiones}

- Se detectó la presencia de Cotylophoron sp en bovinos lecheros de la provincia de Moyobamba, San Martín, con una prevalencia de $55.0 \pm 4.8 \%$.

- No existe diferencia estadística entre la presencia de Cotylophoron sp y el lugar de procedencia, grupo etario y sexo de los animales.

\section{Literatura Citada}

1. Abrous M, Rondelaud D, Dreyfuss G. 1999. Paramphistomum daubneyi and Fasciola hepatica: influence of temperature changes on the shedding of cercariae from dually infected Lymnae atuncatula. Parasitol Res 85: 765-769.
2. Alarcón EP, Velásquez LE. 2009. Descripción morfológica de Cotylophoron cotylophorum (Digenea: Paramphistomidae) hallado en bovinos de Río Negro, Antioquia, Colombia. Rev Colomb Cienc Pecu 22: 168-177.

3. Barriga O. 2002. Las enfermedades parasitarias de los animales domésticos en la América Latina. Santiago: Germinal. 260 p.

4. Dirksen GH, Dieter M, Stober. 2005. Medicina interna y cirugía del bovino. $4^{\mathrm{a}}$ ed. Argentina: Intermédica. $1172 \mathrm{p}$.

5. Eduardo SL. 1982. The taxonomy of the family Paramphistomidae fishoeder, 1901 with special reference to the morphology of species occurring in ruminants. II. Revision of the genus Paramphistomum Fishoeder, 1901. Syst Parasitol 4: 189-238.

6. [INEI] Instituto Nacional de Estadística e Informática. 1994. III Censo Nacional Agropecuario 1994. [Internet]. Disponible en: http://proyectos.inei. gob.pe/bcoCuadros/IIIcenagro.htm

7. Municipalidad de Moyobamba. 2006. Informe de actividades del $1^{\text {er }}$ semestre correspondiente al 2006. [Internet]. Disponible en: http://www.moyobamba. com.pe

8. Paucar S, Chávez A, Casas E, Suárez F. 2010. Prevalencia de fasciolosis y paramfistomosis en el ganado lechero de Oxapampa, Pasco. Rev Inv Vet Perú 21: 87-92. doi: 10.15381/rivep.v21i1.314

9. Paraud C, Gaudin C, Pors I, Chartier C. 2009. Efficacy of oxyclozanide against the rumen fluke Calicophoron daubneyi in experimentally infected goats. Vet J 180: 265-267. doi: 10.1016/ j.tvjl.2008.01.002.

10. Percedo M, Larrmendy R. 1989. Infestación natural de Fossaria cubensis, Pfeiffer, 1839, por estadios larvarios de la familia Paramphistomidae. Rev Cuba Cienc Vet 20: 233-238.

11. Pino LA, Morales G. 1982. Lymnae acubensis Pfeiffer, 1839, hospedador intermediario de Cotylophoron cotylop- 
horum (Fischoeder, 1901) Stiles y Goldberg, 1910, en condiciones naturales. Acta Cient Venezol 33: 57-60.

12. Pinedo R, Chávez A, Casas E, Suárez F, Sánchez N, Huamán H. 2010. Prevalencia de tremátodes de la familia Paramphistomatidae en bovinos del distrito de Yurimaguas, provincia de Alto Amazonas - Loreto. Rev Inv Vet Perú 21: 161-167. doi: 10.15381/rivep. v21i2.132

13. Racioppi O, Lombardero OJ, Moriena RA. 1994. Cotylophoron cotylophorum (Fischoeder, 1901) (Trematoda: Parampohistomidae), nuevo parasito del bovino en Argentina. Rev Med Vet 75: 228-229.

14. Rojas M, Torrel T. 2015. Capítulo 6. Paranfistomidosis. En: Nosoparasitosis de los rumiantes domésticos. Disponible en http://mrojas.perulactea.com/libroparasitismo-de-los-rumiantesdomesticos/libro-nosoparasitosis-de-losrumiantes-domesticos-peruanos/capitulo-6a-paranfistomidosis/

15. Sánchez N, Tantaleán M, Chávez A. Soto A. 2009. Presencia de Cotylophoron cotylophorum (Trematoda, Paramphistomidae) en bovinos de
Loreto, Perú. Rev Peru Biol 16: 141142.

16. Soulsby EJL. 1987. Parasitología y enfermedades parasitarias en los animales domésticos. $7^{\mathrm{a}}$ ed. México: Nueva Ed Interamericana. $823 \mathrm{p}$.

17. Tantaleán M, Martínez R, Juárez D. 1975. Estudio de algunos trematodos del Perú. Rev Per Med Tropical 3-4(1): 4656.

18. Tantaleán M, Sarmiento L, Huiza A. 1992. Digeneos (Trematoda) del Perú. Bol Lima 80: 47-84.

19. Torrel PT. 2008. Paramphistomosis en Cajamarca. Boletín de la Facultad de Ciencias Veterinarias, Universidad Nacional de Cajamarca. $20 \mathrm{p}$.

20. Thrusfield M. 1990. Epidemiología veterinaria. España: Acribia. 339 p.

21. Trigueros A. 2003. Parasitosis gastrointestinal en ovinos Pelibuey en trópico húmedo peruano. En: XXVI Reunión Científica Anual de la Asociación Peruana de Producción Animal. Pucallpa: APPA.

22. Urquhart GM, Armour J, Duncan J, Dunn A. 2001. Parasitología veterinaria. $2^{a}$ ed. Zaragoza: Acribia. 368 p. 